\title{
Surface Treatments of Natural Fibres-A Review: Part 1
}

\author{
Kayode Feyisetan Adekunle ${ }^{1,2}$ \\ ${ }^{1}$ Department of Chemical Engineering, College of Engineering and Engineering Technology, Michael Okpara \\ University of Agriculture, Umudike, Nigeria \\ ${ }^{2}$ Polymer Group, School of Engineering, University of Boras, Boras, Sweden \\ Email: k adekunle@yahoo.co.uk, kayode.adekunle@hb.se
}

Received 3 December 2014; accepted 3 August 2015; published 6 August 2015

Copyright (C) 2015 by author and Scientific Research Publishing Inc.

This work is licensed under the Creative Commons Attribution International License (CC BY).

http://creativecommons.org/licenses/by/4.0/

(c) (i) Open Access

\section{Abstract}

This review is based on the surface treatment of natural fibres which can be used in technical applications. Natural fibres on their own have some draw backs regarding moisture uptake, quality variations, low thermal stability, and poor wettability. Insufficient adhesion between polymer matrix and fibre leads in time to debonding. Overcoming the weaknesses of these natural fibres gave the motivation for this study where physical and chemical methods of modification were considered. Physical methods such as electric discharge and mercerization were reported as well as the chemical methods such as graft copolymerization and treatment with isocyanates, and the results due to these modifications were discussed. This study reveals that natural fibres are good candidates for reinforcement but they have to be suitably treated to improve their properties if they are to be used in technical applications. The various fibre surface treatments actually improve the interfacial adhesion between the fibre surface and the matrix, thereby giving good mechanical properties to the resulted polymer composites.

\section{Keywords}

Natural Fibres, Chemical Modification, Mechanical Properties, Debonding

\section{Introduction}

There are many on going researches to manufacture composites by using natural fibres as reinforcements and polymers from renewable resources as matrices [1]. There is an emerging market for biodegradable polymers which is expected to increase substantially in the coming years [1].

Most polymer matrix composites are made by embedding strong fibres, such as carbon, aramid, glass, or 
natural fibres in a polymer matrix [2] [3]. Depending on their origin, vegetable fibres can be grouped into seed, bast, leaf or fruit qualities [3]. Bast and leaf qualities are so called hard fibres (e.g., flax, jute, and ramie) [4] and are the most used ones [3]. There are also many reports on the use of naturally occurring fibres, including jute, sisal, etc., as reinforcing fibres for polymers [5].

The high strength and modulus of the embedded fibres impart strength and rigidity to the material that surpasses that of the neat polymer [6] [7]. Whereas most composite materials utilize synthetic fibres, such as carbon or glass, in recent years natural fibres have attracted the attention of the composites community as a potential replacement because of the high cost of synthetic fibres [2] [8]. These natural fibres are based on cellulose and offer advantages of biodegradability, low density, nonabrasive nature, and low cost [9] [10].

Significant improvements in mechanical properties have also been achieved due to the reinforcing efficiency of fibres coupled with enhancement through chemical modification in order to promote bonding at the fibrematrix interface [5] [9] [11].

Depending on their origin, natural fibres can be grouped into seed, bast, leaf, and fruit qualities [3] [5]. Bast and leaf quality fibres are the most commonly used in composite applications [3]. Examples of bast fibres include hemp, jute, flax, ramie, and kenaf. Leaf fibres include sisal and banana leaf fibres. Properties for these fibres include excellent tensile strength and modulus, high durability, low bulk density, good moldability, and recyclability.

These natural fibres have an advantage over glass fibres in that they are less expensive, abundantly available from renewable resources, and have a high specific strength [8]. For certain applications, the mechanical properties of natural fibre composites, such as those made from flax or hemp fibre, are not sufficient because of the low strength of these fibres [5] [12]. However, combining natural fibres with stronger synthetic fibres, like glass, could offer an optimum balance between performance and cost.

The properties of the fibre-matrix interface or the interfacial adhesion of the fibre and matrix are of utmost importance to the mechanical properties of composite materials and one way to mechanically improve the interface is thus to achieve efficient chemical bonding between the polymer matrix and the fibre, which could be done in a systematic manner if the surface chemistry of the carbon fibre is understood [7] [13]. However, this chemical bonding can generally be improved by fibre surface treatment, coating of the fibre, addition of a coupling agent, or by tailoring of the chemical properties of the polymer matrix [5] [13]. The physical and chemical methods for the modification of natural fibres were extensively discussed by Bledzki et al. [3].

Natural fibres have many advantages compared to synthetic fibres because they have low density and are recyclable and biodegradable [1] [8] [10]. They are also renewable and have relatively high strength and stiffness [3] [8].

Some disadvantages of natural fibres are attributed to their moisture uptake, quality variations, low thermal stability, and poor wettability [1] [3] [14]. Insufficient adhesion between polymer matrix and fibre leads in time to debonding [4] [14]. There have been many studies as regards the possibilities/ potential of natural fibres as reinforcements for composites and in most cases the results have been favourable in that good stiffness has been achieved in such natural fibre composites [1] [7] [15].

The mechanical properties of natural fibres and their chemical compositions were discussed extensively in this review. The various physical and chemical methods of fibre treatment were discussed and the conclusion was drawn based on the effectiveness of the treatments.

\section{Mechanical Properties of Natural Fibres}

Natural fibres are in general suitable to reinforce plastics thermosets as well as thermoplastics due to their relative high strength and stiffness and low density (Table 1) [16]. The table gives some detail of the mechanical properties of natural fibres.

Natural fibres can be processed in different ways to yield reinforcing elements having different mechanical properties [17]. Fibre properties and fibre structure are influenced by several conditions and varies by area of growth, its climate and the age of the plant. Further, the technical digestion of the fibre is another important factor which determines the structure as well as the characteristics values of the fibres [17]. The tensile strength of natural fibres also depends on the test length of the specimens which is of main importance regarding reinforcing efficiency [17]. Tensile strength of the fibres is also influenced by the refinement of the fibre. 
Table 1. Mechanical properties of natural fibres.

\begin{tabular}{ccc}
\hline Fibre & Density & Tensile strength \\
\hline Cotton & $1.5-1.6$ & $287-597$ \\
Jute & 1.3 & $393-773$ \\
Flax & 1.5 & $345-1035$ \\
Hemp & & 690 \\
Ramie & & $400-938$ \\
Sisal & 1.5 & $511-635$ \\
Coir & 1.2 & 175 \\
Soft wood kraft & 1.5 & 1000 \\
\hline
\end{tabular}

It should also be noted that the hydrophilic nature of all cellulose fibres is a major draw back if the purpose is for reinforcement in plastics. The hydrophilic nature of the natural fibres however influences the overall mechanical properties as well as other physical properties of the fibre itself [17]. Climatic conditions, age and the digestion process influences not only the structure of fibres but also the chemical composition. Except for cotton, the components of natural fibres are cellulose, hemi-cellulose, lignin, pectin, waxes and water soluble substances [17].

\section{Fibre Surface Properties}

Understanding of the fibre surface of natural fibres is very important to the adhesive bonding, natural fibre or wood surface is a complex heterogeneous polymer composed of cellulose, hemi-cellulose and lignin. The surface is influenced by polymer morphology, extractive chemicals and processing conditions [18].

In spite of the limited applicability of these results to the real surface of the natural fibres, the use of the isolated polymers is necessary for estimating the individual wetting characteristics of the various natural fibre components [17].

Toussaint et al. [19], reported about a rapid decrease of the contact angle of water with time for cellulose films (reacted with alkyl ketone dimmer), while for other test liquids such as glycerol, ethylene glycol and diiodomethane a constant contact angle was obtained after 2 - 5 min. Since water has a higher polarity than other liquids, Toussaint et al. discussed the possibility that the decrease in the contact angle is due to specific interactions between water and the cellulose surface allowing water to penetrate into cellulose, causing the cellulose to swell, thus lowering the interfacial free energy and decreasing the contact angle.

Hodgson et al. [20], successfully used the Wilhelmy technique to determine a 'dynamic wettability profile' for wood pulp fibres. He reported about a general decrease of the (advancing) contact angle of distilled water upon repeated immersion because of the hydrophilic nature of this material. The degree to which the value decreases, however, varied significantly for the different types of wood fibres. Pure cellulose fibres exhibited a small $\left(14^{\circ}\right)$ but finite contact angle of water. In general, highly bleached, low-lignin content fibres are more hydrophilic than high yield, high-lignin content fibres.

Liu et al. [21], used dynamic contact angle analysis to characterize the surface energy of differently treaded (heat treated and acetylated) rayon, cotton, and wood fibres. The investigations showed a lower surface energy for the heat treated fibres than for the untreated ones. The surface energy of acetylated wood fibres was 52 dynes $/ \mathrm{cm}$, which was $40 \%$ higher than that of fibres subjected to heat treatment. In the case of acetylated fibres this increase is assigned to the acetyl group polar contribution increase.

The use of different kinds of physical (i.e. corona discharge) and chemical surface treatment methods (coupling agents such as silanes) leads to changes in the surface structure of the fibres as well as to changes in the surface energy.

\section{Fibre Surface Treatment Methods}

To achieve a strong fibre-matrix interfacial adhesion there is an absolute need for the fibres to be treated either 
by using physical or chemical methods. The uses of physical and chemical methods optimize the interface between the fibre and the matrix. These modification methods are of different efficiencies for the adhesion between matrix and fibre [3].

\subsection{Physical Methods}

Physical methods such as stretching, calendaring, thermo treatment and the production of hybrid yarns do not change the chemical composition of the fibres but however they change the structural and surface properties of the fibre and thereby influence the mechanical bonding to polymers [5].

Other ways of treating fibre surface using physical method could be electric discharge such as (corona, cold plasma). Corona treatment is one of the most interesting techniques for surface oxidation activation. It is a process that changes the surface energy of the cellulose fibres and in case of wood surface activation increases the amount of aldehyde groups. Depending on the type and nature of the gases used, a variety of surface modification could be achieved by using cold plasma treatment which will in turn introduce surface crosslinking while surface energy could be increase or decrease and reactive free radicals and groups could be produced [22].

Electric discharge methods are known to be very effective for "non-active" polymer substrates as polystyrene, polyethylene, polypropylene, etc. They are successfully used for cellulose-fibre modification, to decrease the melt viscosity of cellulose-polyethylene composites and improve mechanical properties of cellulose-polypropylene composites [23] [24].

There is also a physical method called mercerization, this is actually an old method used in modifying cellulose fibre, it has been widely used on cotton materials. Mercerization is an alkali treatment of cellulose fibres which depends on the type and concentration of the alkaline solution, its temperature, time of treatment, tension of the material as well as on the additives. It is however possible to use mercerization on natural fibres as well and the optimal conditions ensures the improvement of the tensile properties and absorption characteristics [25].

\subsection{Chemical Methods}

Strongly polarized cellulose fibres are inherently incompatible with hydrophobic polymers. When two materials are incompatible, it is often possible to bring about compatibility by introducing a third material that has properties intermediate between those of the other two. There are several mechanisms of coupling in materials.

The main chemical bonding theory alone is not sufficient, hence the consideration of other concepts appears to be necessary, which include the morphology of the interface, the acid-base reactions at the interface, surface energy and the wetting phenomena.

\subsubsection{Silane Coupling Agents}

These may contribute hydrophilic properties to the interface especially when amino functional silanes, such as epoxies and urethane silanes, are used as primers for reactive polymers. The primer may supply much more amine functionality than can possibly react with the resin at the interphase. Those amines which could not react are hydrophilic and therefore responsible for the poor water resistance of bonds. An effective way to use hydrophilic silanes is to blend them with hydrophobic silanes such as pheniltrimethoxysilane. Mixed siloxane primers also have an improved thermal stability, which is typical for aromatic silicones [26].

\subsubsection{Chemical Coupling}

This is an important chemical modification method which improves the interfacial adhesion. The fibre surface is treated with a compound that forms a bridge of chemical bonds between fibre and matrix.

There are several effective methods of chemical modifications, namely;

1) Graft copolymerization.

2) Treatment with compounds which contain methanol groups.

3) Treatment with isocyanates.

4) Triazine coupling agents.

5) Organosilanes as coupling agents.

For the purpose of this report i would write in details on two of these chemical modifications.

Graft copolymerization: An effective method of chemical modification of natural fibre is graft copolymerization [27]. This reaction is initiated by free radicals of the cellulose molecule. The cellulose is treated with an 
aqueous solution with selected ions and is exposed to a high energy radiation. Then the cellulose molecule cracks and radicals are formed. Afterwards the radical sites of the cellulose are treated with a suitable solution (compatible with the polymer matrix), for example vinyl monomer, acrylonitrile, methyl methacrylate and polystyrene. The resulting co-polymer possesses properties characteristic of both, fibrous cellulose and grafted polymer.

For example, the treatment of cellulose fibres with hot polypropylene-malice anhydride (MAH-PP) copolymers provides covalent bonds across the interface. The mechanism of reaction can be divided into two steps:

1) Activation of the copolymer by heating $\left(t=170^{\circ} \mathrm{C}\right)$ (before fibre treatment) and

2) Esterification of cellulose.

After the treatment the surface energy of the fibres is increased to a level much closer to the surface energy of the matrix. Thus a better wettability and higher interfacial adhesion is obtained. The graft copolymerization method is effective, but complex.

Treatment with isocyanates: The mechanical properties of composites reinforced with wood fibres and PVC or PS as resin can be improved by an isocyanate treatment of those cellulose fibres or the polymer matrix. Polymethylene-polyphenyl-isocyanate (PMPPIC) in pure state or solution in plasticizer can be used. PMPPIC is chemically linked to the cellulose matrix through strong covalent bonds [28].

$$
\mathrm{R}-\mathrm{N}=\mathrm{C}=\mathrm{O}+\mathrm{H}-\mathrm{O}-\text { Cell }-\mathrm{R}-\mathrm{HN}-\mathrm{C}-\mathrm{O}-\text { Cell }
$$

Both PMPPIC and PS contain benzene rings, and their delocalized pie electrons provide strong interactions, so that there is an adhesion between PMPPIC and PS.

Comparing both treatment with silanes and treatment with isocyanates, it is obvious, that the isocyanatic treatment is more effective than the treatment with silane. Equal results are obtained, when PMPPIC is used for the modification of the fibres or polymer matrix [28].

\section{Conclusions}

Natural fibres are good candidates for reinforcement but they have to be suitably treated to elevate their properties if they are to be used in technical applications. Physical and chemical methods can be applied depending of what modification we want to achieve, and since natural fibres have different sources and grown under different climatic conditions, the need to find a suitable method of modification cannot be overemphasized.

The effects of various fibre surface treatments actually improve the interfacial adhesion between the fibre surface and the matrix, thereby giving good mechanical properties to the resulted polymer composites.

\section{References}

[1] Oksman, K., Skrifvars, M. and Selin, J.F. (2003) Natural Fibres as Reinforcement in Polylactic Acid (PLA) Composites. Composites Science \& Technology, 63, 1317-1324. http://dx.doi.org/10.1016/S0266-3538(03)00103-9

[2] Shirikant, N., Lascala, J.J., Can, E., Morye, S.S., Williams, G.I., Palmese, G.R., et al. (2001) Development and Application of Triglyceride-Based Polymers and Composites. Journal of Applied Polymer Science, 82, 703-723. http://dx.doi.org/10.1002/app.1897

[3] Bledzki, A.K., Reihmane, S. and Gassan, J. (1998) Properties and Modification Methods for Vegetable Fibres for Natural Fibre Composites. Journal of Applied Polymer Science, 59, 1329-1336. http://dx.doi.org/10.1002/(SICI)1097-4628(19960222)59:8<1329::AID-APP17>3.0.CO;2-0

[4] Zadorecki, P. and Flodin, P. (1986) Surface Modification of Cellulose Fibres. III. Durability of Cellulose-Polyester Composites under Environmental Ageing. Journal of Applied Polymer Science, 31, 1699-1707. http://dx.doi.org/10.1002/app.1986.070310616

[5] Adekunle, K., Åkesson, D. and Skrifvars, M. (2010) Biobased Composites Prepared by Compression Molding with a Novel Thermoset Resin from Soybean Oil and a Natural-Fiber Reinforcement. Journal of Applied Polymer Science, 116, 1759-1765. http://dx.doi.org/10.1002/app.31634

[6] Hassan, A., Yahya, R., Yahaya, A.H., Tahir, A.R.M. and Hornsby, P.R. (2004) Tensile, Impact and Fiber Length Properties of Injection-Molded Short and Long Glass Fiber-Reinforced Polyamide 6,6 Composites. Journal of Reinforced Plastics and Composites, 23, 969-986. http://dx.doi.org/10.1177/0731684404033960

[7] Luo, S. and Netravali, A.N. (1999) Interfacial and Mechanical Properties of Environment-Friendly "Green” Composites Made from Pineapple Fibers and Poly(hydroxybutyrate-co-valerate) Resin. Journal of Materials Science, 34, 37093719. http://dx.doi.org/10.1023/A:1004659507231 
[8] Dweib, M.A., Hu, B., O’Donnell, A., Shenton, H.W. and Woo, R.P. (2004) All Natural Composite Sandwich Beams for Structural Applications. Composite Structures, 63, 147-157. http://dx.doi.org/10.1016/S0263-8223(03)00143-0

[9] Torres, F.G. and Cubillas, M.L. (2005) Study of the Interfacial Properties of Natural Fibre Reinforced Polyethylene. Polymer Testing, 24, 694-698. http://dx.doi.org/10.1016/j.polymertesting.2005.05.004

[10] Liu, Z.S., Erhan, S.Z. and Calvert, P.D. (2007) Solid Freeform Fabrication of Epoxidized Soybean Oil/Epoxy Composite with Bis or Polyalkyleneamine Curing Agents. Composites Part A, 38, 87-93. http://dx.doi.org/10.1016/j.compositesa.2006.01.009

[11] Hornsby, P.R., Hinrichsen, E. and Tarverdi, K. (1997) Preparation and Properties of Polypropylene Composites Reinforced with Wheat and Flax Straw Fibres: Part I Fibre Characterization. Journal of Materials Science, 32, 443-449. http://dx.doi.org/10.1023/A:1018521920738

[12] Van den Oever, M.J.A., Bos, H.L. and Molenveld, K. (1999) Flax Fibre Physical Structure and Its Effect on Composite Properties: Impact Strength and Thermo-Mechanical Properties. Die Angewandte Makromolekulare Chemie, 272, 71 76. http://dx.doi.org/10.1002/(SICI)1522-9505(19991201)272:1<71::AID-APMC71>3.0.CO;2-R

[13] Gamstedt, E.K., Skrifvars, M., Jacobsen, T.K. and Pyrz, R. (2002) Synthesis of Unsaturated Polyesters for Improved Interfacial Strength in Carbon Fibre Composites. Composites Part A: Applied Science and Manufacturing, 33, 12391252. http://dx.doi.org/10.1016/S1359-835X(02)00077-5

[14] Gassan, J. and Bledzki, A.K. (2000) Possibilities to Improve the Properties of Natural Fiber Reinforced Plastics by Fiber Modification-Jute Polypropylene Composites. Applied Composite Materials, 7, 373-385. http://dx.doi.org/10.1023/A:1026542208108

[15] Herrmann, A.S., Nickel, J. and Riedel, U. (1998) Construction Materials Based upon Biologically Renewable Resources-From Components to Finished Parts. Polymer Degradation and Stability, 59, 251-261. http://dx.doi.org/10.1016/S0141-3910(97)00169-9

[16] Bledzki, A.K. and Gassan, J. (1996) Effect of Coupling Agents on the Moisture Absorption of Natural Fibre Reinforced Plastics. Die Angewandte Makromolekulare Chemie, 236, 129-139. http://dx.doi.org/10.1002/apmc.1996.052360110

[17] Bledzki, A.K. and Gassan, J. (1999) Composites Reinforced with Cellulose Based Fibres. Progress in Polymer Science, 24, 221-274. http://dx.doi.org/10.1016/S0079-6700(98)00018-5

[18] Young, R.A. (1976) Wettability of Wood Pulp Fibers: Applicability of Methodology. Wood and Fiber Science, 8, 120128.

[19] Toussaint, A.F. and Luner, P. (1988) The Wetting Properties of Hydrophobically Modified Cellulose Surfaces. Proceedings of the 10th Cellulose Conference, 29, 1515-1530.

[20] Hodgson, K.T. and Berg, J.C. (1988) Dynamic Wettability Properties of Single Wood Pulp Fibres and Their Relationship to Absorbency. Wood and Fiber Science, 20, 3-17.

[21] Liu, F.P., Wolcott, M.P., Gardner, D.J. and Rials, T.G. (1994) Characterization of the Interface between Cellulosic Fibers and a Thermoplastic Matrix. Composite Interfaces, 2, 419-432.

[22] Sakata, I., Morita, M., Tsuruta, N. and Morita, K. (1993) Activation of Wood Surface by Corona Treatment to Improve Adhesive Bonding. Journal of Applied Polymer Science, 49, 1251-1258. http://dx.doi.org/10.1002/app.1993.070490714

[23] Belgacem, M.N., Bataille, P. and Sapieha, S. (1994) Effect of Corona Modification on the Mechanical Properties of Polypropylene Cellulose Composites. Journal of Applied Polymer Science, 53, 379-385. http://dx.doi.org/10.1002/app.1994.070530401

[24] Dong, S., Sapieha, S. and Schreiber, H.P. (1992) Rheological Properties of Corona Modified Cellulose/Polyethylene Composites. Polymer Engineering \& Science, 32, 1734-1739. http://dx.doi.org/10.1002/pen.760322212

[25] Nevel, T.P. and Zeronian, S.H. (1985) Cellulose Chemistry and Its Applications. Wiley, New York.

[26] Mittal, K.L. (1992) Silanes and Other Coupling Agents. VSP BV, Netherlands.

[27] Ugbolue, S.C.O. (1990) Structure/Property Relationships in Textile Fibres. Journal of the Textile Institute, 20, 41-43. http://dx.doi.org/10.1080/00405169008688950

[28] Maldas, D. and Kokta, B.V. (1989) Improving Adhesion of Wood Fiber with Polystyrene by the Chemical Treatment of Fiber with a Coupling Agent and the Influence on the Mechanical Properties of Composites. Journal of Adhesion Science and Technology, 3, 529-539. http://dx.doi.org/10.1163/156856189X00380 